\title{
Commentary: Potential Mechanism Prediction of Herbal Medicine for Pulmonary Fibrosis Associated With SARS-CoV-2 Infection Based on Network Analysis and Molecular Docking
}

\author{
Congchao Jia ${ }^{1}$, Xinya Peng ${ }^{1}$, Hao Chi ${ }^{1}$ and Gang Tian ${ }^{2 *}$ \\ ${ }^{1}$ Clinical Medical College, Southwest Medical University, Luzhou, China, ${ }^{2}$ Department of Laboratory Medicine, The Affiliated \\ Hospital of Southwest Medical University, Luzhou, China
}

Keywords: COVID-19, network pharmacology, pulmonary fibrosis, molecular docking, Chinese herbal medicine

OPEN ACCESS

Edited by:

Ke-Wu Zeng,

Peking University Health Science Centre, China

Reviewed by:

Chenghai Liu,

Shanghai University of Traditional

Chinese Medicine, China

*Correspondence:

Gang Tian

tiangang@swmu.edu.cn

Specialty section:

This article was submitted to

Ethnopharmacology,

a section of the journal

Frontiers in Pharmacology

Received: 23 September 2021

Accepted: 19 October 2021

Published: 02 November 2021

Citation:

Jia C, Peng X, Chi H and Tian G (2021)

Commentary: Potential Mechanism

Prediction of Herbal Medicine for

Pulmonary Fibrosis Associated With

SARS-CoV-2 Infection Based on

Network Analysis and

Molecular Docking.

Front. Pharmacol. 12:781941.

doi: 10.3389/fphar.2021.781941

\section{Commentary on}

Potential Mechanism Prediction of Herbal Medicine for Pulmonary Fibrosis Associated with SARS-CoV-2 Infection Based on Network Analysis and Molecular Docking

by Jin D., An X., Zhang Y., Zhao S., Duan L., Duan Y., Lian F., and Tong X. (2021). Front Pharmacol. 12:602,218. doi: 10.3389/fphar.2021.602218

The coronavirus disease 2019 (COVID-19) emerged as a global pandemic, and by September 23, 2021 , it brought the cumulative numbers of confirmed cases and deaths globally to more than 230 million and over 4.7 million, respectively. Though the COVID-19 vaccine is available, effectively controlling the rapid spread of COVID-19 and treating the rising number of diagnosed patients has become an urgent worldwide problem to be solved. Thus, it is urgent to develop safe and effective drugs for treating COVID-19. We read the recent article by (Jin et al., 2021) hopefully, which mainly discovered the multi-component, multi-target, multi-pathway mechanism of Convalescent Chinese Prescription (CCP) treatment for COVID-19 pulmonary fibrosis associated with SARS-CoV-2. These findings offer a new perspective on the treatment of COVID-19, but several key issues need to be addressed.

First, there are several questions about the origin of the components and targets of CCP. TCMSP, BATMAN-TCM were all updated earlier than December 2016 and it is questionable whether overly lagging knowledge can facilitate mechanistic investigations of CCP against COVID-19 (Jiang et al., 2020). In addition, Jin et al. used the TCMSP and Swiss TargetPrediction databases for target fishing. It was reported that Swiss TargetPrediction could only achieve at least one correct human target in the top 15 predictions for $>70 \%$ of external compounds (Daina, Michielin, \& Zoete, 2019). Therefore, the component-target interactions predicted by these databases should be validated by molecular dynamics simulation and experiments. Obviously, it is highly implausible because of no experiment in this study.

Secondly, targets of pulmonary fibrosis associated with SARS-CoV-2 are inaccurate. Pulmonary fibrosis is a chronic, progressive process in which healthy tissues are replaced by altered extracellular matrix and alveolar structures are destroyed, resulting in decreased lung compliance, interrupted gas exchange, and respiratory failure and death ultimately (Richeldi, Collard, \& Jones, 2017). We admit 
that pulmonary fibrosis associated with SARS-CoV-2 has some commonalities with pulmonary fibrosis associated with other causes or idiopathic pulmonary fibrosis, but pulmonary fibrosis associated with SARS-CoV-2 also has its characteristics. Unfortunately, Jin et al. did not notice this, and they roughly collected the targets related to pulmonary fibrosis based on the OMIM database, drug bank database, and the DisGeNET database.

Then, in the GO biological process and KEGG pathway enrichment section, the enrichment strategy is unclear and the results are unreliable. Four of the top five of the KEGG pathway (Pathways in cancer, Pancreatic cancer, Bladder cancer, Colorectal cancer) are associated with cancer, which is not relevant to what we are studying.

At the molecular docking part, there are also some problems. Molecular docking is a process through which small molecules are docked into the macromolecular structures for scoring their complementary values at the binding sites (Saikia \& Bordoloi, 2019). The reliability of molecular docking depends on the accuracy of the adopted scoring function, and the scoring function can be used to determine the binding mode and site of a ligand, predict binding affinity and identify the potential drug leads for a given protein target (Li, Fu, \& Zhang, 2019). However, there is no universal scoring algorithm for each molecular docking calculation (Cheng, Li, Li, Liu, \& Wang, 2009; Li et al., 2019; Saikia \& Bordoloi, 2019). Moreover, the docking score is only a relative reference value, and there is no literature supporting that the docking score is greater than three was considered a stable compound binding to the protein.

\section{REFERENCES}

Cheng, T., Li, X., Li, Y., Liu, Z., and Wang, R. (2009). Comparative Assessment of Scoring Functions on a Diverse Test Set. J. Chem. Inf. Model. 49 (4), 1079-1093. doi:10.1021/ci9000053

Daina, A., Michielin, O., and Zoete, V. (2019). SwissTargetPrediction: Updated Data and New Features for Efficient Prediction of Protein Targets of Small Molecules. Nucleic Acids Res. 47 (W1), W357-w364. doi:10.1093/nar/gkz382

Gu, Y. Y., Zhang, M., Cen, H., Wu, Y. F., Lu, Z., Lu, F., et al. (2021). Quercetin as a Potential Treatment for COVID-19-Induced Acute Kidney Injury: Based on Network Pharmacology and Molecular Docking Study. PLoS One 16 (1), e0245209. doi:10.1371/journal.pone.0245209

Huang, Y. F., Bai, C., He, F., Xie, Y., and Zhou, H. (2020). Review on the Potential Action Mechanisms of Chinese Medicines in Treating Coronavirus Disease 2019 (COVID19). Pharmacol. Res. 158, 104939. doi:10.1016/j.phrs.2020.104939

Jiang, S., Cui, Q., Ni, B., Chen, Y., Tan, Y., Chen, W., et al. (2020). Databases for Facilitating Mechanistic Investigations of Traditional Chinese Medicines against COVID-19. Pharmacol. Res. 159, 104989. doi:10.1016/ j.phrs.2020.104989

Jin, D., An, X., Zhang, Y., Zhao, S., Duan, L., Duan, Y., et al. (2021). Potential Mechanism Prediction of Herbal Medicine for Pulmonary Fibrosis Associated with SARS-CoV-2 Infection Based on Network Analysis and Molecular Docking. Front. Pharmacol. 12, 602218. doi:10.3389/fphar.2021.602218

Li, J., Fu, A., and Zhang, L. (2019). An Overview of Scoring Functions Used for Protein-Ligand Interactions in Molecular Docking. Interdiscip. Sci. 11 (2), 320-328. doi:10.1007/s12539-019-00327-w

Niu, W. H., Wu, F., Cao, W. Y., Wu, Z. G., Chao, Y. C., and Liang, C. (2021). Network Pharmacology for the Identification of Phytochemicals in Traditional Chinese Medicine for COVID-19 that May Regulate Interleukin-6. Biosci. Rep. 41 (1), BSR20202583. doi:10.1042/bsr20202583
Finally, the active components identified by the author, such as quercetin, kaempferol, luteolin, are widely distributed in a variety of plants, and a large number of network pharmacology studies have identified quercetin, kaempferol, and luteolin, among others, as candidate molecules for COVID-19 (Huang, Bai, He, Xie, \& Zhou, 2020; Xia et al., 2020; Gu et al., 2021; Niu et al., 2021; Ye et al., 2021). It is highly unreliable. The same molecules being repeatedly found may not indicate an actual result, but instead, they may be due to non-specific interactions by particular molecules. The small molecules above are pan-assay interference compounds, and further experiments are needed to determine whether they have anti-pulmonary fibrosis effects.

COVID-19 remains a global public health problem that places a heavy burden on humanity. The development of anti-COVID19 drugs is urgent, but more attention should be paid to the issues discussed above.

\section{AUTHOR CONTRIBUTIONS}

CJ put forward this view and mainly completed this manuscript. HC and XP discussed the topic and provided some help. GT critically revised the manuscript.

\section{FUNDING}

This study was funded by the Health and Family Planning Commission of Sichuan Province (Grant no. 18PJ104).

Richeldi, L., Collard, H. R., and Jones, M. G. (2017). Idiopathic Pulmonary Fibrosis Lancet 389 (10082), 1941-1952. doi:10.1016/s0140-6736(17)30866-8

Saikia, S., and Bordoloi, M. (2019). Molecular Docking: Challenges, Advances and its Use in Drug Discovery Perspective. Curr. Drug Targets 20 (5), 501-521. doi:10.2174/1389450119666181022153016

Xia, Q. D., Xun, Y., Lu, J. L., Lu, Y. C., Yang, Y. Y., Zhou, P., et al. (2020). Network Pharmacology and Molecular Docking Analyses on Lianhua Qingwen Capsule Indicate Akt1 Is a Potential Target to Treat and Prevent COVID-19. Cell Prolif 53 (12), e12949. doi:10.1111/cpr.12949

Ye, M., Luo, G., Ye, D., She, M., Sun, N., Lu, Y. J., et al. (2021). Network Pharmacology, Molecular Docking Integrated Surface Plasmon Resonance Technology Reveals the Mechanism of Toujie Quwen Granules against Coronavirus Disease 2019 Pneumonia. Phytomedicine 85, 153401. doi:10.1016/j.phymed.2020.153401

Conflict of Interest: The authors declare that the research was conducted in the absence of any commercial or financial relationships that could be construed as a potential conflict of interest.

Publisher's Note: All claims expressed in this article are solely those of the authors and do not necessarily represent those of their affiliated organizations, or those of the publisher, the editors and the reviewers. Any product that may be evaluated in this article, or claim that may be made by its manufacturer, is not guaranteed or endorsed by the publisher.

Copyright (c) 2021 Jia, Peng, Chi and Tian. This is an open-access article distributed under the terms of the Creative Commons Attribution License (CC BY). The use, distribution or reproduction in other forums is permitted, provided the original author(s) and the copyright owner(s) are credited and that the original publication in this journal is cited, in accordance with accepted academic practice. No use, distribution or reproduction is permitted which does not comply with these terms. 